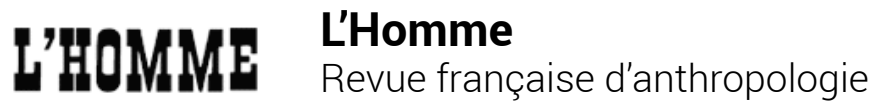

207-208 | 2013

Un miracle créole?

\section{La matrifocalité caribéenne n'est pas un mirage créole}

The Caribbean Matrifocality is not a Creole Mirage

\section{Stéphanie Mulot}

\section{(2) OpenEdition}

Journals

\section{Édition électronique}

URL : http://journals.openedition.org//homme/24691

DOI : 10.4000//homme.24691

ISSN : 1953-8103

Éditeur

Éditions de l'EHESS

\section{Édition imprimée}

Date de publication : 12 novembre 2013

Pagination : 159-191

ISSN : 0439-4216

\section{Référence électronique}

Stéphanie Mulot, «La matrifocalité caribéenne n'est pas un mirage créole », L'Homme [En ligne], 207-208 | 2013, mis en ligne le 05 novembre 2015, consulté le 30 avril 2019. URL : http:// journals.openedition.org/lhomme/24691; DOI : 10.4000/lhomme.24691 


\title{
La matrifocalité caribéenne n'est pas un mirage créole
}

\author{
Stéphanie Mulot
}

À la mémoire de José Balaguette, psychologue $(†$ 2012)

et de François Masure, anthropologue $(† 2013)$

\section{$E$}

ST-IL ENCORE possible, après presqu'un siècle de débat, de parler de matrifocalité caribéenne sans paraître redondant? C'est le défi que nous nous proposons de relever à partir d'une relecture des analyses, anciennes ou contemporaines, consacrées à cette dynamique matrifocale. Car, s'il est bien un sujet qui a largement occupé les recherches sur les sociétés afro-américaines, c'est en effet celui de leur singulier modèle familial que la sociologie anglo-saxonne a qualifié de matrifocal.

La matrifocalité a ainsi fait l'objet d'études menées par des scientifiques originaires des deux côtés de l'Atlantique et de la Caraïbe, ce qui a généré une littérature foisonnante pour tenter de comprendre l'originalité de ce modèle, ainsi que la nature des rapports de genre et le mode de construction des identités sexuelles qui lui sont corrélés au sein de ces sociétés. Associée à la créolisation au même titre que les langues, les systèmes religieux et les modes de représentations du monde, la matrifocalité caribéenne suscita un débat vif et passionné qui succéda à celui sur les familles noires américaines. Novateur au point d'avoir engendré un nouveau concept, celui de "matrifocalité ", ce débat aboutit aussi, paradoxalement, à la négation de cette même forme familiale. Jusqu'à laisser croire que les phénomènes observés pendant des décennies n’étaient que purs mirages... L'évolution de la nature même de la production scientifique contribua largement au changement d'optique à l'œuvre dans l'analyse des modèles familiaux supposés être spécifiquement afro-américains. Cibles, par exemple, de certains travaux de philosophie politique sur l'articulation

J'adresse mes sincères remerciements à Maurice Godelier, Sydney Mintz, Jean-Luc Bonniol et Nadine Lefaucheur, pour leurs conseils avisés et bienveillants. 
des rapports entre sexe, race et classe (Dorlin 2010), les familles matrifocales sont aujourd'hui perçues comme le produit de discours, d'idéologies ou de politiques sexistes et racistes. Elles sont alors niées dans leur réalité empirique, celle des effets des représentations et des interactions sociales inhérentes aux mondes postesclavagistes.

En un siècle, l'analyse des faits sociaux et culturels concernant les populations noires d'une part, la famille et le genre d'autre part, a connu bien des bouleversements, liés à la reconnaissance des droits civiques des Noirs aux États-Unis, à l'accès aux indépendances des pays de la Caraïbe, à la contestation des rapports de domination raciale, coloniale et de genre, ainsi qu'à l'évolution mondiale des formes familiales au gré des contextes économiques. Loin d'être obsolète, le débat sur la matrifocalité nous semble donc, au contraire, mériter une attention nouvelle, à la lumière de ces multiples approches.

\section{Un objet anthropologique mal identifié ?}

La complexité du débat et des analyses concernant les organisations familiales caribéennes tient en partie à leur pluralité et donc, précisément, à la difficulté de les définir. En effet, force est de constater que de multiples formes et structures familiales se côtoient dans l'univers afroaméricain et caribéen, incluant, par exemple, des familles mononucléaires maritales, des familles monoparentales, des familles pluriparentales, des foyers sans enfants ou des ménages multiples. La matrifocalité, qui constitue une dynamique transversale, repose sur l'absence et/ou la disqualification sociale des hommes dans les fonctions paternelles, sur leur présence attendue dans les rôles sexuels hétéronormés et sur la sacralisation sociale de mères qui se disent dévouées pour des enfants qu'elles élèvent parfois sans partenaire stable, mais avec l'aide d'autres femmes de la parentèle. L'expression des femmes antillaises qui proclament "Je suis la mère, je suis le père! » semble résumer cette définition... La diversité des formes familiales au sein desquelles elles s'insèrent a été remarquée, notamment, par Yves Charbit (1987) ou Jacques Cazenave (1997) dans leurs études démographiques, dans nos propres travaux anthropologiques sur la Guadeloupe (2000) et dans les analyses des résultats de l'enquête Genre et violences interpersonnelles en Martinique réalisée par Elizabeth Brown et Nadine Lefaucheur (Lefaucheur \& Brown 2011). Quant à l'origine de cette concomitance de structures familiales, elle avait déjà été rapportée à la fin du XIXe siècle aux États-Unis, par W. E. B. Du Bois (1899 et 1908, cité in Le Dantec-Lowry 2010), qui en situait les fondements dans la période esclavagiste. Cela conforte l'hypothèse de la mise en présence 
de formes antérieures, européennes et africaines, qui ont évolué conjointement, devenant perméables les unes au contact des autres, vers des modèles créoles (Cottias 1990) ${ }^{1}$. Mais cela n'exclut pas pour autant une autre hypothèse, celle d'une dynamique d'adaptation socio-économique applicable à toutes les époques, y compris l'époque contemporaine.

Face à cette diversité des formes familiales, les recherches se sont cependant polarisées sur la plus originale d'entre elles, à savoir l'organisation matrifocale, considérée de surcroît selon une définition bien limitée : la présence, dans un même foyer, de trois générations en lignée maternelle s'occupant des enfants en l'absence de leur père. Son originalité, sa dimension supposée pathogène, l'intérêt anthropologique et sociologique suscité par la découverte de nouvelles formes familiales, la preuve qu'elle apportait d'une production culturelle noire nouvelle - ou d'un héritage africain transgénérationnel - et la source de revendications qu'elle représente pour certains acteurs en présence qui s'en disent les victimes, constituent probablement les raisons d'une telle focalisation sur cette dynamique familiale particulière...

Pour mieux comprendre cette singularité, il nous semble nécessaire de considérer la matrifocalité non plus suivant une lecture verticale (la présence de plusieurs générations de femmes dans une même maisonnée en l'absence d'hommes dans les rôles paternels), mais plutôt suivant une lecture horizontale et transversale. Une telle posture méthodologique permettra, en effet, de saisir la nature des relations intrafamiliales, les modes de construction des identités de genre qui s'y jouent et les liens entre les dynamiques familiales et sociales ${ }^{2}$. Dès lors, émergeront les modes de représentations des identités féminines et masculines, maternelles et paternelles, sociales et "raciales " qui caractérisent le modèle matrifocal, ainsi que l'ensemble des rapports sociaux de sexe et leurs liens avec l'organisation sociale globale. Loin d'être cantonnées aux structures monoparentales, ces représentations sont présentes dans l'ensemble de la société.

Nous nous proposons donc de revenir sur les différentes approches - anciennes ou récentes - de la recherche sur les familles noires, la matrifocalité caribéenne et ses corollaires (le genre et la sexualité), en nous interrogeant à chaque fois sur leur pertinence dans le contexte antillais contemporain. Il est assez frappant de constater la pluralité d'études sur

1. Myriam Cottias (cf. infra) a montré que la créolisation des familles africaines et européennes se fait lorsqu'elles tendent progressivement vers un modèle commun qui reconjugue des fonctionnements et dynamiques de chacune. La fécondité, la natalité et la nuptialité des différents groupes présents sur le sol antillais évoluent selon les contacts de population et l'adaptation au milieu.

2. Ce fut l'objet, que nous ne développerons pas ici, de notre thèse de doctorat à laquelle nous renvoyons les lecteurs pour plus de précisions (Mulot 2000). 
la question et la difficulté permanente à comprendre le fonctionnement même de cet objet. Soulignons enfin que, si des observations sur le terrain ont été réalisées par nos collègues anglophones et hispanophones sur les îles de la Caraïbe, un nombre limité de recherches anthropologiques, fondées sur une ethnographie longue, rigoureuse et monographique de familles antillaises, concerne les îles françaises. Par ailleurs, les travaux des chercheurs caribéens ont été peu pris en compte jusqu'à présent, du fait de leur diffusion encore faible en France.

Outre que la critique féministe l'oriente singulièrement aujourd'hui, le débat français sur la matrifocalité est actuellement porté par plusieurs disciplines. La sociologie ou la philosophie politique en nient complètement l'existence, au motif de la dénonciation de l'idéologie raciste, sexiste, bourgeoise, ethnocentrique et religieuse qui aurait présidé au façonnement de la notion et des analyses dont elle a fait l'objet (Giraud 1999; Dorlin 2010). Les études historiques des organisations familiales antillaises durant la période esclavagiste ou post-abolitionniste (Cottias 1990 ; Gautier 1985; Chivallon 2004) montrent la concomitance de plusieurs modèles matrifocaux et patriarcaux. Les approches statistiques de la famille ou des comportements sexuels, qui insistent sur la part du pluripartenariat hétérosexuel, sont fondamentalement intéressantes, mais ne disent rien de la dynamique relationnelle des acteurs concernés (Cazenave 1997 ; Giraud et al. 1994; Halfen 2006). Les recherches menées dans des institutions juridiques, sociales ou psychiatriques auprès de familles en difficulté et auprès du personnel soignant (André 1987 ; Lesel 1996 ; Rolle-Romana 1999; Massé 2008) peuvent, par le biais du recrutement de leur échantillon, entraîner une focalisation sur la dimension pathogène du phénomène observé. Celles menées dans une approche qualitative mêlant à la fois monographies, entretiens et observations sont rares (Attias-Donfut \& Lapierre 1997 ; Mulot 2000 ; Lefaucheur \& Brown 2011 ; Lefaucheur \& Mulot 2012) - plus encore, celles mêlant approches quantitatives et qualitatives -, ou ont été réalisées ailleurs que sur le terrain antillais, notamment dans l'Hexagone ou en Europe auprès de migrants caribéens (Pourette 2006; Condon \& Byron 2008).

Force est donc de constater que la perception des fonctionnements internes aux familles antillaises est soit biaisée, soit limitée. Alors qu’il serait important de croiser les approches quantitatives et qualitatives sur des terrains différents, il apparaît que les résultats des recherches restent plutôt fragmentaires ou servent à nier l'existence d'une forme familiale singulière, présentée comme un mythe qui se déroberait face aux chiffres (Chivallon 2004), en particulier les statistiques sur les foyers antillais. Or, 
si l'utilité de ces statistiques ne fait aucun doute pour relativiser la part d'un phénomène en soulignant sa dimension plurielle, l'unité de mesure proposée par l'INSEE (le foyer, le nombre de personnes y résidant, les liens de parenté entre elles) ne suffit pourtant pas à décrire la réalité des liens entre les membres d'une famille, surtout si l'acception locale de celle-ci dépasse la notion de foyer nucléaire ${ }^{3}$. Le caractère ethnocentrique d'un tel outil oublie, en effet, que les relations familiales (et, parmi elles, l'éducation des enfants, les rapports hommes-femmes, les liens intergénérationnels) ne se cantonnent pas à l'espace du foyer. Au contraire, et notamment dans les cas de familles non cohabitantes ou de familles recomposées, la dynamique relationnelle se joue à l'extérieur, dans un "en-dehors» que les pratiques sociales et sexuelles antillaises ont investi fortement depuis longtemps. Et ce sont ces dynamiques internes et non les structures familiales que nous proposons d'identifier avec cette relecture des caractéristiques de la matrifocalité et de ses enjeux.

Nous choisissons donc, pour l'instant, de définir la matrifocalité comme un mode relationnel - et non comme une structure - déterminant la construction du genre et l'expérience d'une parentalité qui sacralise les mères et disqualifie les pères. Cette dynamique reste marquée par des représentations héritées de la période esclavagiste.

\section{La matrifocalité, un objet créole? Le débat fondateur sur l'origine des familles noires, ou la fausse route initiale}

Avant même que le terme "matrifocalité " ne soit inventé, des études sur les « familles noires » s'étaient développées en tentant de comprendre la difficulté des hommes noirs à être présents dans les rôles paternels, difficulté alors perçue comme une carence morale et sociale majeure. Ces organisations familiales pouvaient-elles être considérées comme de véritables familles? Quelle définition de la famille fallait-il en retenir? L’origine raciale, la couleur, la catégorie sociale des personnes étaient-elles les causes de ces différences entre familles noires et blanches? Une telle absence de morale était-elle due à une absence de culture et d'éducation quasiment constitutive de l'identité " raciale », physique et biologique des " Noirs », ou était-elle le résultat de contingences historiques, économiques ou sociales?

3. L'accès au logement individuel favorisé par les politiques publiques a largement contribué à faire diminuer le nombre de personnes ainsi que le nombre de générations cohabitant au sein du même foyer. Cette "nucléarisation" des unités résidentielles suffit-elle à remettre en question toute la dynamique matrifocale? 
Les premières études sur les familles noires aux États-Unis, réalisées dès la fin du XIX ${ }^{\mathrm{e}}$ siècle, étaient fortement marquées par un contexte raciste qui ne pouvait accorder aux Noirs une once d'humanité. Selon Hélène Le Dantec-Lowry, c'est le travail de W. E. B. Du Bois qui, pour la première fois, témoigna d'une opposition à ce déterminisme racial ambiant, pour favoriser au contraire la prise en compte de facteurs sociaux et environnementaux. Il constatait " une "destruction de la vie familiale répandue et précoce" (Du Bois 1899: 66) [qu'il attribuait] à une "morale relâchée" et imput[ait] ces problèmes en grande partie au régime esclavagiste (Ibid. : 67) » (Le Dantec-Lowry 2010 : 39). «L'influence du passé est évidente dans les habitudes des mariages faciles et des séparations aisées ", avançait-il de façon audacieuse en 1908 (Du Bois 1969 [1908] : 130, cité in Le Dantec-Lowry 2010 : 39). Il soulignait l'existence de liens familiaux multiples chez les esclaves et l'importance des critères socio-économiques et culturels qui permirent à une élite noire favorisée de développer le modèle conjugal et marital.

Quelques années plus tard, c'est Edward Franklin Frazier qui s'attela à prouver l'importance des déterminismes historiques et sociogéographiques dans la difficulté des Noirs à constituer des familles stables. Reprenant l'argument de Du Bois, et utilisant les apports de Robert Park et de l'école de Chicago qui se constituait, il insista sur les trois moments fondateurs qui aboutirent à la déstabilisation des familles noires: la traite et l'esclavage $\mathrm{du} \mathrm{XV}^{\mathrm{e}}$ au XIX $\mathrm{X}^{\mathrm{e}}$ siècle; l'émancipation et la perte de repères moraux de la fin du XIX ${ }^{e}$ siècle; l'exode rural, la Grande Migration et l'urbanisation du début du XXe siècle. Déstructuration, démoralisation et désorganisation sont donc les processus analysés par Frazier pour expliquer la situation des familles noires urbaines et contrecarrer l'hypothèse de carences raciales, qui risquait, selon lui, de légitimer les discriminations et les ségrégations envers les Noirs dans les villes américaines. Il insista, au contraire, sur les effets extrêmement délétères de trois facteurs sociaux découlant des trois moments fondateurs qu'il avait définis: l'asservissement esclavagiste fut un véritable rouleau compresseur qui empêcha la survie de toute forme familiale antérieure; l'émancipation laissa les esclaves dans un abandon moral incompatible avec toute prise de responsabilité familiale; enfin, un nouveau déplacement acheva de déstabiliser ces familles noires, en renforçant leur précarité. Frazier, dans cette nouvelle lecture des déterminismes structurels et non plus raciaux, proposa ainsi une vision de l'esclavage comme événement durablement aliénant et déstructurant : "Il est fort probable que jamais auparavant dans l'histoire un peuple n'ait été aussi dépouillé de son héritage social que les Noirs qui furent débarqués en Amérique» (1973 [1939] : 20, 
ma traduction). Cette vision s'est retrouvée durant des décennies dans de nombreux travaux, notamment ceux des chercheurs et écrivains soutenant l'idée de sociétés krazé ("écrasées»), marquées par l'aliénation, l'asservissement, la dépossession, la privation ou l'écrasement culturels.

Une troisième thèse, défendue dans les années 1940 par Melville J. Herskovits, anthropologue blanc élève de Franz Boas, vint orienter différemment le débat, à la fois vers la Caraïbe et vers l'Afrique. Selon cet auteur, les comportements des Noirs des Amériques provenaient d'un héritage africain reconfiguré en Amérique. À partir d'études menées sur les cultures noires à Harlem et à la Jamaïque, il développa l'hypothèse diffusionniste de résurgences africaines dans le Nouveau Monde, et entreprit de répertorier dans plusieurs régions américaines des échelles d'africanismes, selon que les traits culturels observés étaient plus ou moins proches d'une forme africaine supposée originelle. Son ouvrage de 1941, The Myth of the Negro Past, a ouvert la voie à une série de travaux désormais afro-américanistes, insistant sur la capacité des esclaves à maintenir dans leurs institutions (famille, religion, langage, économie...) un espace culturel traditionnel, en s'opposant peu ou prou à l'entreprise de colonisation, soit par le marronnage, soit par des formes de résistance culturelle collective. Les africanismes furent même perçus comme le résultat de résistances intentionnelles ayant permis la transposition, intacte ou réinterprétée, d'éléments d'une culture africaine originelle. Cette perspective diffusionniste et culturaliste suppose donc des esclaves soucieux de conservation culturelle, mais également des traits africains qui auraient servi de matrice pour revisiter les normes et valeurs de la société blanche. Ainsi la fréquence du pluripartenariat sexuel et les unions consensuelles seraient les réinterprétations par les systèmes polygamiques africains des normes de mariage européennes.

Les enjeux de ce débat étaient avant tout d'ordres politique, économique et idéologique. Il s'agissait de déterminer si les Noirs étaient capables d'assimiler la culture américaine, ses règles morales, religieuses et sociales et s'ils pourraient un jour être considérés comme des citoyens à part entière. À l'inverse, le prisme de la "bestialité noire» présent dans les discours politiques et rapporté par Herbert Gutman (1972) devait-il les maintenir hors des rangs de la citoyenneté ? Dans ce débat sur la primauté des déterminismes raciaux, sociaux ou culturels, Du Bois insista donc sur la nécessité de revenir aux fondements esclavagistes, Frazier souligna les effets destructeurs et aliénants de l'entreprise coloniale et de la vie urbaine sur les modes de vie des anciens esclaves, tandis que Herskovits préféra retenir les survivances des cultures africaines en Amérique. 
Au cours du $\mathrm{XX}^{\mathrm{e}}$ siècle, nombreux sont les penseurs à avoir suivi cette dernière hypothèse de résurgences africaines, parfois au point de développer des théories panafricanistes ou afrocentristes, comme l'a souligné Christine Chivallon (2004) à propos, notamment, de Mervyn Alleyne. Or, si chez Herskovits la survivance de traits africains ne remet pas en cause le fait que la culture noire a su aussi assimiler les éléments nécessaires à son intégration dans la société américaine, le discours contemporain semble donner la priorité aux aspects revendicatifs et conservateurs de cette fidélité culturelle... Ainsi, pour ce qui concerne les Antilles françaises, n'est-il pas rare de voir rappelé dans les débats, travaux scientifiques, ou dans les revendications politiques, que l'organisation familiale antillaise serait un héritage direct de la culture africaine et de la résistance d'esclaves qualifiés aujourd'hui de Marrons. En les reliant directement à une vision - souvent caricaturale et erronée - de l'Afrique dont les systèmes de parenté auraient tous été matrilinéaires, et les formes d'alliance polygamiques, cet argument cherche à justifier, en les valorisant, les modes de vie antillais. L'enjeu, derrière de tels arguments, est de se positionner soit comme descendants d'esclaves, soit comme "afrodescendants» et, ainsi, de se reconnaître comme porteurs d'une culture meurtrie par la colonisation, dans un cas, ou sauvée par la résistance des esclaves, dans l'autre, et ce, in fine, dans l'intention de demander éventuellement réparation pour les crimes commis et les préjudices subis, notamment au niveau des institutions familiales (Mulot 2010) ${ }^{4}$.

La teneur de ce débat sur l'origine des formes familiales "noires " nous semble présenter plusieurs écueils importants: une vision souvent ethnicisée et racialisée de ces familles selon une dichotomie Noir/Blanc, une importance trop grande accordée à des continuités et façonnements culturels plutôt qu'à l'analyse des dynamiques contemporaines, des références à un ailleurs originel (africain) occultant les fondements créoles de ces pratiques sociales et une identification des acteurs contemporains à des ancêtres mythifiés. Seule la créolisation permit de sortir de cette fausse route.

4. Si les revendications du collectif guadeloupéen LKP, lors de la grève générale de 2009, ont laissé complètement de côté la question des relations familiales, le Comité Marche du 23 mai 1998 (CM98) entend, pour sa part, demander à la République de prendre la "mesure des dysfonctionnements familiaux hérités de l'esclavage " en soutenant la création, dans l'Hexagone, du premier "Centre d'aide aux familles matrifocales et monoparentales». Cité dans la déclaration du Comité Marche du 23 mai de 2008 et consultable sur internet [http://halleyjc.blog.lemonde.fr/2008/ 11/14/declaration-de-la-delegation-antillaise-recue-le-13-novembre-par-la-presidence-de-larepublique/]. 


\section{L’apport de la théorie de la créolisation}

La thèse de la continuité africaine coexiste, par ailleurs, chez certains auteurs comme Roger Bastide (1973 [1967]), avec l'image d'un esclave adaptateur et interprète des institutions dont il a hérité en fonction du contexte, de l'environnement et du cadre social - celui de l'esclavage et de la plantation - dans lesquels il évolue. Mais, elle s'est pleinement exprimée dans ce qu'il est aujourd'hui convenu d'appeler la "théorie de la créolisation ». Prenant position dans la polémique sur l'origine des cultures noires américaines bien après les débats fondateurs, Sidney Mintz et Richard Price avançaient : "Aucun groupe, même en y étant préparé, ne peut transférer ses modes de vie, ses croyances et ses valeurs intacts, d'un lieu à un autre " (1976 : 40, ma traduction). Revenant précisément sur la question de l'origine historique ou biologique des faits culturels, Sidney Mintz avait remarqué que la confusion entre traits culturels et traits physiques ne permettait pas de sortir d'une vision raciale. Or, assenait-il, « il faut répéter avec insistance que certains Afro-Américains sont phénotypiquement blancs " (1971:4, ma traduction). Cette affirmation remet donc en cause les travaux précédents, en permettant de sortir d'une dichotomie raciale et, de surcroît, mieux saisir le principe de créolisation qui rend compte de la construction culturelle de ces sociétés afro-américaines. Comme cet auteur a pu le développer dans ses différents travaux, les cultures créoles sont nées, non pas de l'évitement ou de la partition sociale entre les Noirs et les Blancs, mais bien de l'interpénétration lente et progressive de leurs deux univers, à la fois aux niveaux social, culturel et biologique. Il ne s'agit donc ni d'un syncrétisme ni d'une fusion. Pour ma part, j'appelle « créolisation" ce processus progressif d'interpénétration culturelle, par lequel deux groupes en présence, dans un contexte de forte domination sociale et politique de l'un par l'autre, se sont transformés en incorporant, petit à petit et par à-coups, au fil de négociations symboliques, idéelles et matérielles, âpres voire sanglantes, des éléments de la culture de l'autre.

Cette perspective permet de sortir d'une vaine archéologie des phénomènes créoles qui, dans une "épistémologie de la vérification" (Scott 1997), rechercherait les traces et preuves de l'origine fondatrice des faits observés. Comme l'affirmait Sidney Mintz, «le contenu des cultures afroaméricaines dans cet hémisphère prend toute sa signification dans l'usage que les Afro-Américains en font, et non dans le fait que ses origines soient ou ne soient pas africaines " (1970: 13, ma traduction). La question principale que cette anthropologie afro-américaine devrait donc aborder est celle du sens et des valeurs que les Américains et les Caribéens ont donnés à des pratiques culturelles nouvelles: nouvelles puisque sorties de leur 
contexte originel ou nouvelles puisque créées formellement de toutes pièces. Dans les deux cas, ce sens et ces valeurs dépendent d'une représentation du monde entièrement forgée, quant à elle, dans l'univers colonial de la plantation, dès lors radicalement inédite et éloignée des origines. Le contexte de la plantation est bel et bien un lieu de vie ou de survie, un lieu de domination et de rapports sociaux, politiques et sexuels, ainsi qu'un lieu de production symbolique, fondant la perception et la culture de ceux qui ne seront plus totalement des Africains, ni des Européens.

Myriam Cottias (1990) a montré que la population blanche s'est elle-même créolisée, pas seulement physiquement mais également à travers ses comportements familiaux et sexuels. De même, le taux de natalité et le nombre d'enfants par femme-esclave ont sensiblement rejoint ceux constatés chez les Blancs créoles au fil des décennies et des siècles (Ibid.). Ainsi, progressivement, les valeurs et modèles coloniaux pouvant influencer les esclaves ne sont plus européens, mais fondamentalement créoles. Réciproquement, les conduites des Blancs créoles ne s'inspirent plus des seules formes européennes, elles subissent une modification lente mais indéniable. Le modèle du pluripartenariat antillais ne peut donc plus, dans son vécu créole, être appréhendé d'un strict point de vue dichotomique: ce n'est ni une forme de polygamie africaine aux fondements politiques, ni la seule traduction d'une pratique européenne et bourgeoise de l'adultère empreinte de machisme ; c'est une forme de relation plurielle qui se décline différemment selon les sexes et les stratégies de promotion sociale (fierté, distinction ou condamnation sociales).

\section{La place de l'alliance dans l'organisation matrifocale}

\section{La culture de la pénurie et l'affaiblissement de l'alliance}

Raymond T. Smith proposa, pour la première fois, en 1956, dans son ouvrage The Negro Family in British Guyana, le terme spécifique de "matrifocalité " pour désigner cette adaptation socio-économique à la société de plantation. Préférant ce terme à ceux de " matricentralité » ou de «matriarcat", il insista sur le fait que, dans ce modèle, ce sont les mères qui sont au centre des relations familiales, ainsi axées sur des relations de filiation plus que sur des relations d'alliance. Mettant avant tout l'accent, dans son analyse des classes populaires défavorisées, sur les critères socioéconomiques (pauvreté, pénurie, chômage des hommes et des femmes, migrations des pères), Raymond T. Smith conclut que, dans les «familles noires", les femmes auraient besoin d'un compagnon pour élever les enfants en bas âge, mais s'en passeraient plus facilement à mesure qu'ils grandissent et participent aux frais ménagers. Dès lors que la présence 
d'un homme à la tête du foyer n'est plus une nécessité économique, ledit foyer deviendrait du coup féminin, maternel et non conjugal, donc matrifocal. Cette contrainte de l'éloignement serait accentuée par la situation de précarité professionnelle que connaissent les hommes, par ailleurs obligés de se déplacer vers les villes pour trouver du travail. Précarité, migrations, veuvage accroittraient ainsi la part des foyers matrifocaux.

Raymond T. Smith empruntait à Meyer Fortes la conviction que "ce qui est indispensable, c'est le lien parental, et pour ceci toute forme de cohabitation permettant la procréation est suffisante" (cité in André [1987 : 22]). Cette approche fondamentale, accordant la préférence à la filiation et non à l'alliance, a profondément marqué la façon d'appréhender les familles matrifocales et le caractère subsidiaire de la paternité. Ici, les relations conjugales ne sont pas considérées d'un point de vue affectif et/ou éducatif, mais du strict point de vue matériel et économique. Les relations de couple se résumeraient essentiellement à des échanges économico-sexuels.

La précarité professionnelle et économique serait donc à la base de l'« instabilité familiale " dite matrifocale en concentrant les relations familiales autour du personnage maternel devenu central. Le débat scientifique n'envisage plus la famille caribéenne "noire» dans une dialectique historique de la résistance ou de l'anéantissement, mais, cette fois, dans une dialectique sociologique de l'adaptation ou de la déviance à la norme maritale. C'est pourquoi plusieurs chercheurs vont même désigner comme matrifocales des familles issues d'autres aires culturelles, en soulignant la similitude des conséquences liées à la précarité ou à la migration masculine sur la structuration familiale. Ainsi, que ce soient dans certains quartiers de Londres ou en Écosse selon Raymond T. Smith, dans la société javanaise ou en Amérique centrale selon Nancy L. Gonzales (1970), chez les Nayars de l'Inde du Sud selon Ralph Kunstadter (1963), ou bien encore dans les familles occidentales ayant connu des situations de ruptures ou de décès, des formes de matrifocalité s'observeraient dans ces "foyers de consanguins" (autour d'une femme et de ses descendants), qui se développeraient de façon similaire en fonction de critères économiques défavorables. Ces questions sont également au centre des travaux de Michael G. Smith (1962) et de Edith Clarke (1966 [1957]) à la même période, sur les Antilles anglophones, qui précisent le rôle central de la mère dans l'organisation matrifocale et le caractère périphérique de la paternité.

Une telle vision de la matrifocalité est à l'origine d'une confusion majeure qui fausse aujourd'hui encore le débat scientifique. Même si nous devons à Raymond T. Smith d'avoir inventé ce terme et d'avoir fait le lien entre familles matrifocales et organisations sociales souvent associées à 
une culture de la pauvreté - et non plus à leur seule origine africaine -, il apparaît que cela a pu entretenir un amalgame entre matrifocalité, familles pauvres (donc souvent noires) et monoparentalité. Cette dernière notion n'était pas encore utilisée dans les années 1950 et 1960, mais son émergence dans l'analyse des effets de la baisse de la nuptialité et de la hausse des décohabitations conjugales dans les familles européennes et occidentales a favorisé cette confusion toujours présente entre monoparentalité et matrifocalité. C'est la raison essentielle pour laquelle cette dernière est aujourd'hui considérée comme un mythe par les quantitativistes, qui ne décomptent que les structures monoparentales.

Or, il nous semble que nous sommes face à deux processus différents. Si la matrifocalité peut en effet trouver sa place dans des foyers monoparentaux, à l'inverse, la monoparentalité ne suffit pas à la définir. En cela, nos conclusions sont différentes de celles de Raymond T. Smith, puisque les critères que nous retenons englobent, au-delà d'éventuels aspects économiques, les modes de construction des identités sexuelles et parentales, les modes de représentations du monde qui assignent aux individus des identités individuelles et collectives influencées par l'héritage spécifique de l'esclavage et, surtout, une dynamique relationnelle particulière entre les sexes, les générations et les groupes racialisés. Selon nous, la matrifocalité caribéenne est le fruit des processus de créolisation sociale, culturelle et identitaire, et ne peut se résumer à une forme d'adaptation économique que n'importe quelle société connaîtrait sous la configuration monoparentale. En outre, le consensus sur l'incompétence des pères reste le trait caractéristique de la matrifocalité, davantage que sa facette monoparentale. Enfin, il apparaît que si la matrifocalité est née dans un contexte économique singulier, elle se manifeste aujourd'hui dans d'autres registres que celui de l'économie.

Cependant, Raymond T. Smith et ses contemporains ont permis de distinguer la matrifocalité du matriarcat, d'en montrer les fondements économiques, la force d'adaptation et la cohérence interne. Car, l'accent mis sur la dimension économique ne doit pas être dénigré pour autant. Aux États-Unis, la pauvreté des familles noires et la précarisation des hommes semblent aussi être propices au développement de la matrifocalité, même si elle n'y est pas désignée comme telle (Bourgois 2001). Aux Antilles françaises, l'organisation matrifocale, qui suppose des modes de rapports intergénérationnels, des formes de solidarités intrafamiliales pour compenser les faibles moyens financiers, des circulations d'enfants dans la parentèle pour aider aux tâches domestiques ou pour leur éducation, se développerait bel et bien en lien avec une "mémoire de la pauvreté» (Attias-Donfut \& Lapierre 1997 : 115). De plus, les données 
démographiques montrent que la nuptialité varie nettement d'une catégorie socio-professionnelle à une autre, en fonction du capital à transmettre et à consolider (propriété foncière, entreprise, patronyme, etc.), et donc de stratégies de promotion sociale. C'est ainsi que Christine Chivallon (2004) a pu noter que chez les propriétaires terriens de la Martinique le modèle conjugal était nucléaire et patrifocal. La nécessité pour les propriétaires terriens de disposer d'une organisation domestique compatible avec le travail d'exploitation agricole a probablement encouragé la présence de femmes sur les domaines, à la fois dans leurs foyers et en tant que travailleuses agricoles, ce qui a dû favoriser la nuptialité et la répartition sexuée des tâches. Pour autant, aujourd'hui encore, les ménages les plus favorisés ne sont pas exempts de fonctionnements matrifocaux, même dans des foyers maritaux. En outre, la matrifocalité ne signifie absolument pas que ceux qui l'incarnent dénigrent la valeur du mariage.

\section{Le mariage comme référentiel partagé}

Michael G. Smith est le premier, en 1962, à mettre au cœur de son analyse la relation homme-femme malgré sa forme extra-résidentielle. Il insiste sur le fait que, dans les familles antillaises, les enfants savent toujours qui est leur père, même s'il ne vit pas sous le même toit. Le père est en effet désigné par la mère et existe dans l'univers proche ou lointain des enfants, en leur rendant des visites plus ou moins occasionnelles. Ces familles antillaises se définissent donc moins comme des familles avec un seul parent, que par leurs relations complexes entre parents et enfants. Le sociologue distingue alors trois types d'unions qui se succèdent dans les parcours matrimoniaux : en phase initiale, une relation de type extrarésidentielle dite "visiting union", le concubinage à l'âge adulte et le mariage qui viendrait entériner la maturité du couple 5 . Pourtant, comme l'indiquera plus tard Jacques André, «d'une manière générale, l'homme n'est pas visiteur ou concubin ou marié, il est concubin ou mari et visiteur» (1987 : 31). La concomitance éventuelle ou recherchée entre les trois formes conjugales rend caduque toute opposition entre elles. Elles ne s'excluent pas; elles peuvent même être conjointes. De surcroît, la distinction de ces trois étapes correspond à une différence de niveau social : l'union extra-résidentielle serait particulière aux classes populaires, le concubinage aux classes moyennes et le mariage aux classes supérieures.

5. Cette analyse nous permet de souligner l'extrême modernité des sociétés créoles, dont les structures familiales (bien que le sens en fût différent...) donnaient déjà un avant-goût de ce que sont devenues les relations de couple dans les sociétés industrialisées de la fin du XXe siècle. 
Or, le terrain antillais nous montre que, au-delà des clivages sociaux, le mariage est considéré comme un idéal dans toutes les catégories. Ce sont plutôt les modalités de sa concrétisation qui varient d'une classe à l'autre, en fonction des capacités économiques et sociales de chacun, et de la propension à vouloir adhérer à la norme morale ou, au contraire, à la transgresser ${ }^{6}$.

Chez plusieurs auteurs, la vie maritale apparaît comme une norme, un aboutissement vers lequel tendent les hommes et les femmes, même s'ils mettent aussi en évidence la concrétisation de relations familiales en dehors des liens maritaux. Déjà, dans les années 1960, le débat entre déviance et adaptation à cette norme conjugale opposa les chercheurs, notamment William Goode et Hyman Rodman. Ce dernier avait judicieusement noté que la supériorité numérique des unions consensuelles en milieu populaire ne signifiait pas, comme le premier le prétendait, que cette catégorie n’idéalisait pas le mariage. Au contraire, pour le cas de Trinidad, il remarquait que la classe populaire avait la capacité d'adapter son système de valeurs en fonction des situations vécues, ce qu'il nomma "l'étirement des valeurs dans la classe populaire ", c'est-à-dire un idéal du mariage et, conjointement, une acceptation des unions consensuelles qui, loin d'être opposés, constituaient « deux types de modèles conjugaux acceptables" (Rodman 1963 : 274).

Cette prise en compte de la valeur accordée à l'alliance, qui permet de faire émerger des significations nouvelles, de nature créole, fut à la fois pertinente et sous-estimée. Guy Dubreuil (1965) abonda dans le même sens en mettant en exergue un système de valeurs commun à l'ensemble des formes familiales, matrifocales ou autres, rencontrées en Martinique, quels que soient les milieux sociaux. La hiérarchie qui organise ce système étant "souple et polyvalente", il observait une dynamique évolutive de ces formes familiales les unes vers les autres. Aujourd'hui encore, la force attractive de la vie conjugale ou du mariage peut se lire dans le comportement de certaines mères célibataires qui, utilisant leur grossesse comme moyen de pression, cherchent à obtenir du père de l'enfant qu'il devienne un conjoint ou un mari. Elle peut également se lire dans l'importance relative des mariages tardifs, contractés après 50 ans, qui nous renseigne sur la valeur morale de ce sacrement que les individus souhaitent recevoir avant leur mort, non seulement pour faire montre d'une stabilité sociale enfin acquise, mais aussi, en ce qui concerne les croyants, pour sauver leur

6. D'autre part, à situation économique semblable, certains groupes culturels tels les «Indiens », ont longtemps maintenu des relations matrimoniales patriarcales, ce qui constitue un contreexemple. 
âme avant le Jugement dernier. En outre, le refus des femmes de divorcer malgré des situations conjugales pénibles témoigne notamment de leur souci de préserver leur statut marital et de continuer à jouir du prestige de porter le même nom de famille que leurs enfants et que le père de ces derniers. Une juge aux affaires matrimoniales nous rapportait le discours de femmes entamant une procédure de divorce, qui se résignaient à « rendre » son nom au mari : «An ka bay non ay (Je lui rends son nom)». Cette question du nom de famille, acquis, porté, transmis et qu'il faut rendre le cas échéant, vient souligner les enjeux pouvant exister autour de la légitimité des unions et des naissances. Même si les familles s'accommodent généralement du fait que les enfants d'une même fratrie utérine puissent ne pas tous porter le même nom de famille, la différence de statut entre enfants légitimes et non légitimes, ces " enfants-dehors » 7 , est souvent la cause de profondes discordes, notamment au moment des héritages...

\section{Un mode créole de construction des identités sexuelles}

\section{Le double standard dans l'éducation et la socialisation des jeunes}

La matrifocalité se lit aussi à travers la succession de formes conjugales, qui reposent sur la pratique d'un pluripartenariat successif et/ou simultané, usuellement rapportée, dans les études caribéanistes, aux normes concernant l'éducation sexuelle des garçons et des filles. Les comportements sexuels sont liés à deux critères régulateurs des conduites masculine et féminine : le critère de la réputation caractérise plutôt la conduite des garçons, quand celui de la respectabilité ressortit davantage à celle des filles. Ce double standard a été mis en évidence par Peter Wilson $(1969,1973)$ dans ses travaux sur l'île anglophone de Providencia, où il a produit les premières analyses d'importance sur la construction des identités sexuelles dans la Caraïbe. La respectabilité y est présentée comme un ensemble de valeurs et de normes héritées de l'organisation coloniale, qui distingue les individus, et particulièrement les femmes, selon leurs capacités à les suivre et les reproduire. La moralité, la religion, l'éducation constituent les piliers de cette respectabilité revendiquée par les classes moyennes et supérieures comme critères de distinction. À l'inverse, l'impératif de réputation,

7. L'expression créole "Ti moun déwô" insiste sur l'extériorité de l'enfant non reconnu, vivant avec sa mère, par rapport aux enfants reconnus et vivant avec leur père. Il apparaît que certaines femmes souhaitent aujourd'hui que leurs enfants ne soient pas reconnus afin, disent-elles, de pouvoir bénéficier de prestations sociales particulières en tant que parent isolé. La reconnaissance ou non des enfants devient un sujet de conflit important entre les parents. 
produit de la communauté des pairs, concerne les hommes. La réputation se fonde sur des valeurs "indigènes ", locales, construites à partir de l'adaptation aux conditions de vie dans l'univers caribéen (post-)esclavagiste : la défense de l'honneur, du respect, le machisme, la sagesse, l'éloquence, voire certains talents artistiques (musique, danse...).

Afficher ses conquêtes, son pouvoir de séduction, narrer ses exploits sexuels ou savoir se mettre en valeur en travaillant son apparence, son langage ou sa gestuelle sont donc autant de codes comportementaux que les hommes antillais doivent savoir maîtriser. Ils leur permettent de gagner leur place au sein de la communauté des pairs, mais aussi d'être reconnus par les femmes qui, elles-mêmes, peuvent apprécier d'avoir affaire à de "vrais hommes" (ou par les mères qui s'enorgueillissent de voir leurs fils devenir des «coqs»). Ce thème a largement été repris dans les études francophones, notamment par Jacques André (1987), Michel Giraud (1999), Christiane Bougerol (2002) ou dans nos propres travaux (Mulot 2000, 2009). La permanence de ce double standard, qui n'est pas sans rappeler des formes universelles de différenciation de genre dans l'éducation des enfants, interroge sur ses éventuels effets sur la structuration familiale. Est-ce du fait de leur matrifocalité que les familles incitent les garçons à investir l'espace public, et les filles à se cantonner dans l'espace domestique? Ou bien est-ce parce qu'il existe des communautés de pairs (masculines ou féminines) fonctionnant comme des agents de socialisation très influents que les individus s'engagent différemment dans les rôles domestiques et contribuent, de la sorte, à la matrifocalisation des familles?

\section{La socialisation par les pairs : faute de père, et de mère ?}

Dans la Caraïbe, l'Université des West Indies a produit une série de travaux sur la question du genre, au point qu'un département "Gender and Development Studies » y a été créé, depuis les années 1990. Des recherches et colloques importants, dont plusieurs ouvrages se sont fait l'écho (Reddock 2004 ; Lewis 2003 ; Chevannes 1999), ont plus particulièrement porté sur la thématique de l'identité masculine caribéenne, à travers l'analyse des différences entre l'éducation des filles et celle des garçons, des violences envers les femmes, de l'homophobie, des diverses formes de délinquance auxquelles se livrent les jeunes hommes et de la difficulté rémanente des hommes à être présents dans l'espace conjugal. Un courant féministe novateur a parcouru l'ensemble de la réflexion sociologique, montrant que la condition féminine est loin d'être enviable dans cet univers caribéen, même si les femmes assument aujourd'hui plus souvent que les hommes des responsabilités sociales. 
Revenant sur la place des groupes de pairs dans la construction des identités sexuelles masculines à La Jamaïque, Barry Chevannes (1999) a montré que ces groupes constituent pour les hommes une puissante force de socialisation interne et mutuelle, et de contrôle social, souvent plus influente que l'autorité des adultes et des parents. S'agit-il là de la compensation d'un vide de pouvoir (power vacuum) favorable à des conduites à risques multiples, comme le prétendent David Plummer et ses collègues (Plummer, McLean \& Simpson 2008) ? Il apparaît dans tous les cas, et notre ethnographie le soulignait aussi pour la Guadeloupe, que c'est bien au sein de ces groupes de pairs que se définissent, par l'intermédiaire des aînés qui les transmettent aux cadets, les valeurs, les représentations, les critères et les contours de la domination masculine, excluant par là même les conduites jugées intolérables (homosexualité, conduites efféminées, fragilité, sensibilité, fidélité sexuelle...). Les femmes ne désapprouvent pas ces impératifs; au contraire, elles peuvent aussi les encourager pour tester la virilité des hommes qu'elles rencontrent.

Si les groupes de pairs féminins semblent moins visibles, les activités domestiques, de voisinage, de commérage sont pourtant le lieu d'une forte socialisation féminine où les aînées, souvent des mères, dressent elles aussi les cadres d'une féminité respectable (discrétion, fidélité, dévouement pour les enfants...) et d'une féminité condamnable (liberté et pluripartenariat sexuels, fréquentation des espaces masculins et nocturnes, délaissement des tâches familiales...). Par un effet de contrôle croisé, le regard des hommes sur les femmes et celui des femmes sur les hommes viennent renforcer les interdits dans chacune de ces communautés (Mulot 2008). En outre, ces groupes subissent des influences extérieures, par le biais des médias et de la diffusion de la pornographie (Mulot 2009), ou encore par celui des migrations et du tourisme, et par l'accès aux études qui ouvrent de nouveaux horizons et confrontent à d'autres normes de genre (Pourette 2006). Ainsi, dans le contexte antillais francophone, les échanges fréquents avec l'Hexagone contribuent-ils à la diffusion de modèles de rapports sociaux de sexes sensiblement moins inégalitaires, même si ces modèles ont toujours bien du mal à échapper au rouleau compresseur du contrôle des groupes de pairs.

L'évolution contemporaine des normes de masculinité et féminité mériterait d'être plus longuement analysée, notamment à l'aune des modes de racialisation du genre, comme l'a fait David Murray (1999) pour la masculinité martiniquaise. L'enquête ${ }^{8}$ Genre et violences interpersonnelles

8. Réalisée à la fin des années 2000 par Nadine Lefaucheur du Centre universitaire des Antilles et de la Guyane et par Elisabeth Brown de l'Université de Paris Panthéon-Sorbonne. 
à la Martinique (cf. Lefaucheur 2011) permet à ce propos de souligner que des forces contradictoires orientent la masculinité aujourd'hui : d'un côté, le souci de ne pas reproduire les conduites des hommes des générations précédentes, en assumant vie conjugale et éducation des enfants, et, de l'autre, dans une frange de la population jeune, cette nécessité toujours présente de faire preuve de liberté, de construire sa réputation sexuelle, d'affirmer une virilité agressive, voire de commettre des actes de délinquance afin de se faire respecter dans la culture de la rue (Lefaucheur \& Mulot 2012). Différentes enquêtes qualitatives font état de ce paradoxe de l'identité masculine antillaise, entre des injonctions à la domination masculine et des doutes sur les fondements de cette virilité. À partir des résultats de l'enquête Genre et violences interpersonnelles à la Martinique, Joëlle Kabile et Clara Palmiste (2012) ont avancé l'hypothèse que l'éducation des garçons, pauvre en reconnaissance affective et morale de la part des parents, principalement de la mère, serait l'une des causes d'un manque d'estime et de confiance en soi, expliquerait la violence qui les caractérise (que ce soit envers les femmes ou envers d'autres hommes) et leur difficulté à investir pleinement toute vie sociale, familiale et citoyenne.

De leur côté, si les femmes semblent de moins en moins accepter le pluripartenariat masculin, la résignation ou la peur face à la violence physique dont font preuve leurs compagnons à leur égard limitent encore les velléités d'émancipation. En outre, et ce point est central dans le dispositif matrifocal, l'impossibilité d'échapper à une vision de la féminité en termes de respectabilité et de sacralisation maternelle empêche toute remise en question des rapports sociaux de sexe. Condamnées à être soit respectables, soit considérées comme des "salopes " ${ }^{9}$, c'est-à-dire soit comme des mères sacralisées, soit comme des mauvaises mères, les femmes ne peuvent guère assumer publiquement, souhaiter intimement ou vivre pleinement une sexualité plus libre, ou ne serait-ce qu'une conjugalité moins rivée aux tâches maternelles. Ainsi le pluripartenariat féminin reste-t-il l'un des plus grands tabous de la société antillaise, même si certaines femmes adoptent le rôle de maîtresses d'hommes pluripartenaires, au cœur de rapports économico-sexuels. Quant à la figure de la femme-mère sacrifiée, elle demeure l'un des piliers de l'imaginaire matrifocal.

9. L'insulte «salope " reste en créole l'une des plus souvent utilisées pour désigner et rabaisser publiquement les femmes qui se risqueraient à afficher plusieurs partenaires. 


\section{Repenser les cadres psychiques, imaginaires et politiques de la matrifocalité \\ Analyser l'imaginaire familial antillais}

Comprendre les représentations imaginaires antillaises de la féminité et de la masculinité, de la maternité et de la paternité nous semble essentiel pour saisir les enjeux de la dynamique matrifocale. Cela n'est possible que par une longue pratique ethnologique du quotidien des Antillais, une analyse de la littérature orale et écrite, ou par une lecture psychoanalytique des relations familiales et conjugales. C'est à ce dernier point que se sont attelés des psychiatres, psychologues et psychanalystes antillais et métropolitains depuis Frantz Fanon et son analyse du désir en contexte antillais (1952), « en questionnant la distribution des relations affectives et en distinguant les personnes psychiques des personnes réelles " (André 1987 : 40).

"L'investigation psychanalytique conduit à inverser les termes du raisonnement: la position précède la fonction, il faut une mère déjà constituée - comme personne psychique - pour que puisse être investie l'activité correspondante [...]. La matrifocalité au sens où nous l'entendons est une matrifocalité sexuelle. La mère focale n'est pas une conséquence, elle est au principe" (Ibid. : 37-38).

L'apport d'une telle démarche est multiple. Elle permet de faire émerger des représentations transmises au sein de l'espace familial et social, et qui constituent autant d'images des rôles sexuels et parentaux que les individus sont amenés ou non à jouer. La matrifocalité apparaît alors comme une organisation construite sur des fonctions psychiques et des représentations qui déterminent l'identité sexuelle, familiale, sociale, voire "raciale» des individus. La mère focale et le père absent sont des images construites et élaborées au fil d'une histoire particulière, et réactivées dans chaque famille par l'intermédiaire de l'éducation des enfants. Ainsi, la mère focale préexiste dans le désir des sujets antillais et détermine leurs actes. Elle est au centre du dispositif matrifocal non plus seulement en tant que conséquence (d'une histoire et d'une socio-économie données), mais aussi en tant que cause.

Les membres du Centre antillais de recherche et d'études (CARE) ont produit une littérature essentielle sur ces représentations, s’interrogeant sur la place des femmes et des hommes noirs dans l'imaginaire de l'esclavage, ou sur la figure du maître. Car la trame de cet imaginaire de l'ancestralité antillaise s'est formée à partir de la position médiane de ce dernier, exerçant un pouvoir absolu sur les esclaves, de la destitution à son profit de leur droit à la paternité et de la place nodale occupée par les femmes 
noires sur les habitations, fréquentant l'univers domestique du maître et susceptibles d'en connaître les failles et les rouages. La captation du pouvoir par le maître, dépossédant les hommes noirs, se serait accompagnée d'une captation du désir des femmes noires pour leur dominateur, dans une stratégie d'émancipation et de promotion sociales pour elles et pour leur descendance métisse. Dépossédés par les maîtres, dédaignés par les femmes, les hommes esclaves n'auraient alors pu investir ni la sphère domestique ni les fonctions paternelles, plus soucieux de prouver par ailleurs l'existence ou la survivance de leur virilité.

Retrouvant ces représentations dans les discours contemporains, les auteurs analysent le lien singulier, parfois de type incestueux, qui unit une mère à ses enfants dans le dispositif matrifocal. Véritable "mère gigogne " (Lesel 1995), entretenant avec sa progéniture une relation fusionnelle et de dette imprescriptible qui empêche la rupture de l'" amarre " maternelle (André 1987), la mère apparaît comme actrice de l'évacuation du père de la fonction éducative. Disqualifié, oblitéré parfois dans le discours tenu aux enfants, enjoint de ne se réaliser que dans les activités sexuelles et économiques, l'homme s'inquiète de savoir s'il est bien l'objet du désir des femmes, ces femmes-mères qu'il peut percevoir comme castratrices. Le pluripartenariat masculin pourrait alors avoir une fonction anxiolytique..., bien que peu réparatrice...

Nous avons eu l'occasion d'analyser ailleurs l'influence du mythe du "viol fondateur" sur la construction des identités sexuelles contemporaines (Mulot 2007). L'imaginaire antillais se représente, en effet, l'origine de la société créole et métissée dans le viol des premières ancêtres africaines par les marins et les colons européens blancs, sous le regard impuissant des hommes noirs (Glissant 1981). C'est donc à partir de cette scène fondatrice que se sont construites ces identités, forcément meurtries et figées dans un passé fantasmatique. Les femmes en sortiraient paradoxalement grandies, pour leur résistance et leur rôle de passeuses d'une culture africaine préservée, tandis que les hommes noirs se trouveraient marqués au sceau de l'impuissance à secourir leurs femmes et de l'incapacité à être les pères de leurs enfants, au profit du maître. Pourtant, ce mythe fondé sur le non-consentement de la femme noire face au violeur blanc propose une vision des rapports de sexe et de race bien différente de celle que l'histoire contemporaine a pu véhiculer, notamment autour des stratégies qu'auraient déployées ces femmes noires pour séduire les hommes blancs (Cottias 2001). Ce non-consentement apporterait, en effet, la preuve de leur fidélité affective envers les hommes noirs et garantirait à ces derniers leur statut le plus précieux : rester l'objet du désir des femmes, être des sujets désirants et désirés. 
Le mythe du «viol fondateur» institue donc trois piliers de la matrifocalité : la diffraction d'une paternité ne pouvant être totalement assumée ni par les esclaves ni par les maîtres; la sacralisation de femmes-mères victimisées, qu'aucun homme ne peut véritablement soutenir et à qui une dette devra être payée à chaque génération; la castration symbolique des hommes noirs, désormais condamnés à faire continuellement la preuve de leur puissance sexuelle.

\section{Contester une vision coloniale de la famille et de la sexualité}

En France, les travaux des historiens, en l'occurrence des historiennes, ont ouvert les débats féministes qui s'attachaient à dénoncer la condition féminine dans la société coloniale. Arlette Gautier pour la Guadeloupe, Myriam Cottias pour la Martinique ont ainsi montré que l'idée d'une société dominée par les femmes était erronée : leur présence à la tête de familles monoparentales ne devait pas masquer leur marginalisation sur la scène publique, ni leur soumission dans la sphère conjugale. Insistant sur les formes de domination sexuelle et raciale que les femmes de couleur ont subies, et analysant les moyens qu'elles se sont donnés pour y résister, ces auteures ont défini les familles antillaises comme le lieu où les femmes esclaves ont tenté de construire leur autonomie, leur reconnaissance et, plus tard, leur citoyenneté, à l'aide de plusieurs stratégies d'émancipation, tout en restant contraintes par les pressions patriarcales du Code noir et du Code civil napoléonien. La précision est de taille : le dispositif familial s'est en effet mis en place aux Antilles dans un cadre légal qui était d'ordre patriarcal, reconnaissant le pouvoir domestique uniquement aux hommes. Or, jusqu'à l'abolition de 1848 , seuls les maîtres pouvaient jouir de ce pouvoir et seul l'accès à la citoyenneté permit aux anciens esclaves de l'exercer au sein de leurs familles. Dans ce contexte, Myriam Cottias (2002) formula l'hypothèse que les anciennes esclaves, considérées comme des citoyennes mineures, adoptèrent les principes de respectabilité et de légitimité conjugale et sociale, tout en refusant, pour certaines, le mariage afin de préserver la part d'autonomie qu'elles étaient chèrement parvenues à acquérir au fil de siècles de servitude. Il est probable que certains anciens esclaves aient eu, de leur côté, des difficultés à investir ce pouvoir qu'on leur reconnaissait tardivement, et auquel les femmes, dans la sphère domestique, se sont opposées tant bien que mal. Aujourd'hui encore, nous l'avons observé, ce partage du pouvoir et de l'autorité domestiques reste le nœud de nombreux conflits conjugaux et l'une des veines de l'organisation matrifocale. En outre, les politiques familiales jugées plus défavorables aux familles antillaises qu'aux familles européennes ont probablement contribué au maintien de foyers matrifocaux (Gautier 1986). 
Bien après les travaux fondamentaux de Frantz Fanon (1952) sur l'aliénation du désir chez les colonisés, Michel Giraud (1999) a analysé les représentations de la sexualité des hommes et des femmes noirs dans l'imaginaire européen. À l'appui de ses recherches, notamment sur les comportements sexuels aux Antilles (Giraud et al. 1994), le sociologue interprète la focalisation et la stigmatisation du "multipartenariat" hétérosexuel caribéen, telles qu'elles ont été opérées par les chercheurs européens, comme une déformation liée à un regard ethnocentriste, chrétien, moraliste et familialiste. Et cette stigmatisation serait d'autant plus forte que l'écart par rapport au modèle européen idéal de la famille - bourgeoise, conjugale et maritale - devait être souligné pour mieux valoriser ce dernier.

Plus récemment, les travaux de la philosophe Elsa Dorlin ont à leur tour apporté un éclairage nouveau, dans l'optique postcolonialiste du Black feminism (2007). Elle se propose de comprendre les comportements sociaux à partir d'une lecture des discours scientifiques et idéologiques, en s'attachant à décrire les processus de racialisation et de sexualisation opérés par la médecine et l'anthropologie coloniales. Selon cette auteure, le système de l'esclavage reposait non seulement sur le contrôle des corps et de la sexualité des esclaves, mais aussi sur une inversion de genre. Cela passait, d'un côté, par une effémination et une bestialisation des hommes noirs, qui, malgré leur sexualité animale auraient été incapables de contenter leurs partenaires, décrites comme insatiables et lubriques. Perçues comme ayant des capacités de résistance, une force physique et une solidité qui justifiaient le sort qui leur était réservé, les femmes noires étaient, de leur côté, virilisées et érotisées (Dorlin \& Parris 2006). Leurs qualités de reproductrices auraient été utilisées pour la fabrique d'esclaves, alors que leur «maternité monstrueuse" (Dorlin 2010: 72), amorale, expliquait l'anormalité des familles noires. Ce croisement des genres - des hommes symboliquement castrés et efféminés et des femmes virilisées - justifiait la domination raciale et la violence sexuelle que les Blancs exerçaient alors sur eux.

Toujours selon Elsa Dorlin, le mythe du «matriarcat noir" s'est développé d'abord aux États-Unis au $\mathrm{XX}^{\mathrm{e}}$ siècle, notamment avec le rapport Moynihan (1965), pour condamner les prétendus travers des familles noires, décrites comme pathogènes du fait d'un "pouvoir castrateur des femmes noires sur les hommes noirs" (Dorlin 2010 : 74). Citant les travaux de Patricia Hill Collins, elle montre que ce mythe, nourri des stéréotypes de la Bad Black Mother et de la Welfare Queen qui ne vivrait que de prestations sociales, sert à justifier le refus de l'État 
américain de payer pour les déviances d'une population considérée comme responsable et coupable de ses propres maux. Dans le contexte français et antillais contemporain, de telles suspicions à l'égard des foyers monoparentaux et matrifocaux sont également fréquentes. Les parents, et particulièrement les mères, sont soupçonnés de préférer au cadre moral du modèle patriarcal l'avantage des prestations familiales ${ }^{10}$.

Cependant, il convient de s'interroger sur la portée réelle des discours sociaux et scientifiques qu'analyse Elsa Dorlin. Ont-ils véritablement influencé (et comment ?) les pratiques et modes de pensée des individus au quotidien, ou se sont-ils plutôt fait l'écho de comportements qui les précédaient d'un ou deux siècles? Plusieurs idéologies sont en fait à l'œuvre: celle en fonction de laquelle se sont établies, autrefois, des pratiques sociales; celle qui a produit a posteriori les discours qui les analysaient; celle qui, aujourd'hui, interprète les pratiques du passé en fonction de représentations du présent; ou celle qui préside à la formation de pratiques contemporaines souvent coupées du passé, mais légitimées selon une continuité historique réinventée. Notre regard sur les pratiques matrimoniales et sexuelles est donc le produit d'un ensemble de strates idéologiques plus ou moins performatives. Il nous semble que les comportements contemporains trouvent dans ces idéologies des modes de légitimation, de renforcement ou de compensation qui peuvent encore aujourd'hui constituer une entrave à l'évolution des rapports sociaux de sexe et de race. Le fait, par exemple, que certain(e)s soient convaincu(e)s d'avoir pour ancêtres des femmes violées, voire des hommes castrés, n'est pas sans conséquence : la victimisation des femmes, la condamnation des mauvaises mères et des femmes pluripartenaires, ainsi que l'obsession des hommes à prouver leur puissance sexuelle et physique peuvent trouver leurs racines, non pas tant dans l'histoire en elle-même, mais dans l'interprétation de celle-ci au fil de littératures, de discours sociaux et "scientifiques" fortement idéologisés. C'est donc un travail sur l'imaginaire ainsi que sur l'ensemble des représentations du genre et de la parentalité qui permettra d'élucider les rapports sociaux contemporains, plutôt que la mise en cause directe d'une histoire dont le récit reste encore partiel.

10. La condamnation des mères qui profiteraient des services sociaux est courante dans le contexte antillais, où les foyers monoparentaux ont toujours posé problème aux acteurs socio-éducatifs et politiques. 


\section{Questionner autrement la matrifocalité via le genre aujourd'hui}

\section{Repenser le genre caribéen au masculin...}

Si la remise en question des figures de la féminité et de la maternité semble se heurter à un tabou profond dans la société antillaise, une autre approche de la masculinité caractérise, par ailleurs, les travaux de nos collègues caribéens ${ }^{11}$. Prenant le contrepied de l'accusation courante pointant les faiblesses masculines, Errol Miller, sociologue jamaïcain, développe l'hypothèse que les hommes auraient été victimes de marginalisations éducatives, sociales ou familiales (Miller 1986)... Observant que, dans la Caraïbe anglophone, les hommes sont maintenant moins présents que les femmes dans les universités, occupent moins de postes de décision, assument moins de responsabilités familiales, mais sont plus impliqués dans des prises de risque diverses, telles que la délinquance ou autres actes de violence, il s'interroge sur l'effet de l'émancipation féminine sur le vécu et l'identité des hommes. Il attribue cette marginalisation à deux facteurs : le recul des hommes noirs, fragilisés par cette présence de plus en plus marquée des femmes noires sur la scène familiale, éducative et sociale, d'une part; et les formes de domination que les femmes noires peuvent exercer sur les hommes en utilisant éventuellement des alliés blancs, d'autre part.

Si cette thèse a fait grand bruit dans la Caraïbe anglophone, c'est que le discours de Miller stigmatisait les femmes qui, selon lui, auraient pu, dans l'histoire, s'allier avec des hommes blancs à des fins de promotion sociale, et avoir ainsi pris part à l'éviction des hommes noirs et à leur dépréciation socioraciale. Cette image de femmes noires "complices" du maître a choqué un lectorat féministe qui, par l'intermédiaire du mouvement du Black feminism, dénonçait, à l'inverse, la domination masculine blanche et noire: sexuelle, raciale et sociale. S'inspirant des travaux de Patricia Hill Collins notamment, ce féminisme tendait plutôt à souligner les formes de résistance des femmes noires durant et après l'esclavage, la façon dont elles ont su exploiter leur connaissance du monde blanc, celui du maître, qui les opprimait.

L'histoire montre en effet, notamment pour le cas des Antilles françaises, que certaines femmes noires ont su développer des stratégies d'émancipation par le métissage, par les alliances, par la mobilité sociale et préférer les privilèges du pouvoir des Blancs. Fritz Gracchus l'avait bien résumé : «Entre le pénis de l'homme noir et le phallus de l'homme blanc,

11. Citons, notamment, Linden Lewis (2007) et Rhoda Reddock (2004). 
la femme a fait son choix" (1980: 181). Tenant parfois une position dominante et respectée dans la société, elles n'en demeuraient pas moins sous le coup d'une accusation permanente de traitrise, incompatible avec l'image courante de femmes-victimes et de "mère-courage", ou avec le fantasme contemporain d'une "communauté " noire soudée, solidaire et sans faille, unie en un front racial, social et politique bâti pour mieux faire face à l'oppresseur blanc ${ }^{12}$.

\section{... et au féminin}

Au-delà du débat ouvert par Errol Miller, les chercheurs caribéens se sont intéressés à la complexité de la construction et de l'expérience des masculinités dans la Caraïbe. Un très riche ouvrage collectif dirigé par Rhoda Reddock (2004) témoigne de ce renouveau qui visait notamment à questionner les outils théoriques et les données empiriques pour analyser la situation des hommes caribéens. Dans un chapitre particulièrement heuristique, Patricia Mohammed, s’inscrivant dans une épistémologie féministe postmoderne, propose de penser, conjointement et de façon articulée, masculinité et féminité en sortant des dichotomies et stéréotypes usuels :

« [...] l'homme blanc puissant et dominant ; l'homme noir vivant dans la promiscuité et l'irresponsabilité ; l'homme indien violent [...] et excessivement patriarcal dans ses relations conjugales; la femme blanche fragile et faible, soit riche soit objet d'un mépris social ; la femme de couleur objet sexuel ; la femme noire forte et indépendante, dirigeant un foyer matrifocal et méfiante envers les hommes; la femme indienne créature passive et subalterne, etc. " (2004: 45 , ma traduction).

Dans une approche sociohistorique, reprenant un précédent travail de 1995, elle met en relief les effets de la colonisation sur la négociation des rapports de pouvoir, entre les hommes, entre les femmes, et entre les hommes et les femmes, au sein d'un même groupe ethnique et entre les groupes ethniques blancs, créoles et indiens. La déconstruction de la notion de patriarcat, comme outil d'analyse des familles caribéennes, lui parait donc indispensable pour comprendre les variations et les remises en question des rapports de domination entre hommes et femmes. Elle aboutit ainsi à la distinction de plusieurs formes de patriarcat, construites en fonction des luttes politiques et symboliques qui les ont traversées : le patriarcat blanc d'origine européenne; le patriarcat créole qui a été supplanté par l'hégémonie de la domination masculine blanche et a

12. Les figures de la Métisse et de la Mulâtresse illustrent tout à fait cette suspicion d'alliances inquiétantes (Mulot 2008). 
vu l'émergence des femmes dans les rapports de pouvoir; et le patriarcat indo-caribéen qui lutte contre sa propre créolisation et contre la prise de pouvoir des femmes. Elle invite alors à l'analyse des inter-dépendances dans les constructions de la masculinité et de la féminité dans la Caraïbe.

Une telle proposition s'inscrit à la suite des travaux américains sur la nécessité de penser la consubstantialité des rapports de genre et de race au sein de la communauté africaine-américaine et de ses mouvements féministes et sociaux. Autour de Cornel West (2003) et de Bell Hooks (1995) notamment, un consensus émerge sur la nécessité de ne plus considérer la famille patriarcale blanche ou noire comme référent unique, mais plutôt de choisir un modèle égalitaire, comme le souligne Hélène Charlery (2007: 84). Le patriarcat, en tant qu'il renforce la domination masculine, ne peut être la solution aux difficultés, violences et limites rencontrées par les hommes, les femmes et les familles noirs, contrairement à ce qu'envisageaient les mouvements virilistes noirs, comme la Nation of Islam. L'enjeu fondamental est de repenser la masculinité, la féminité et le patriarcat, dans une réflexion qui se décentre de la sexualité et de la famille pour articuler les problèmes de genre, de race et de classe.

\section{Penser les violences masculines et féminines dans les relations familiales}

L'enquête Genre et violences interpersonnelles à la Martinique nous incite aussi à penser la masculinité et le patriarcat sous un angle nouveau, en considérant effectivement les négociations des rapports de pouvoir entre hommes et femmes. Au-delà de l'importante violence subie par les filles et les conjointes que révèle l'enquête, plusieurs résultats nous obligent à envisager aussi la position dominante qu'occupent certaines femmes dans différentes relations: les mères, les grands-mères, les rivales, mais aussi les conjointes, dans des relations d'éducation, de jalousie, de conflits (Lefaucheur 2012; Mulot 2012). Or, la violence des femmes mérite également d'être analysée, non pas pour minimiser le fait que nombre d'entre elles sont victimes par ailleurs de violences graves voire mortelles, mais pour souligner que la négociation des rapports de pouvoir n'est pas unilatérale et que des paramètres sociaux et culturels peuvent venir remettre en question la seule domination masculine. En l'occurrence, la croyance partagée dans la population antillaise en une autorité féminine, et plus particulièrement maternelle (que d'aucuns nomment matriarcale ou matrifocale), interdit la remise en question - sacrilège - du discours des mères et favorise leurs doléances et la contestation féminine des comportements masculins. Plusieurs profils de femmes habituellement peu visibles émergent ainsi de l'enquête Genre et violences... : celles qui 
dénigrent leur compagnon, celles qui malmènent leurs enfants en les privant éventuellement de tendresse et d'attention, celles qui provoquent les conflits conjugaux à leurs risques et périls, celles qui refusent de reconnaître une place de parent aux pères de leurs enfants... Ces différents profils reflètent la permanence de relations matrifocales dont il faut mesurer les effets et les conséquences pour les hommes et les femmes, plutôt qu'en chercher l'origine.

\section{Concilier analyse féministe, critique du patriarcat et reconnaissance de la matrifocalité}

La principale difficulté dans l'analyse des dynamiques relationnelles des familles antillaises tient, on le comprendra, au souci de concilier une posture féministe et critique, dénonçant ainsi le patriarcat et les différentes formes de domination (sociale, raciale et sexuelle, notamment) dont les femmes sont victimes, avec les données socio-anthropologiques, empiriques et cliniques qui révèlent la complexité des comportements féminins et maternels. "Comment réussir à évoquer la réalité du terrain antillais et rester fidèle à ses convictions féministes?", se demandait une psychologue militante travaillant en Martinique depuis de nombreuses années.

La notion de "patriarcat fissuré » que j'ai développée récemment (Mulot 2011) pourrait permettre de dépasser cette difficulté, en reconnaissant la concomitance des deux modèles observés : patriarcat et matrifocalité. En effet, elle renvoie à la fois aux rapports de domination de sexe imprégnés de l'idéologie patriarcale transmise par les cultures originelles des Européens et des Africains (et des Indiens), mais aussi aux limites de ce patriarcat dans le contexte post-esclavagiste, qui s'est construit à partir de l'éclatement et de la fissuration profonde du pouvoir, de la puissance et de l'autorité des hommes noirs esclaves et qui a donné aux femmes noires, par le travail, la monoparentalité et l'éducation, la capacité de contester la domination masculine noire et blanche. Créole, fissuré et éclaté, ce patriarcat antillais ne saurait cependant être aujourd'hui restauré en se fondant sur le renforcement de la domination masculine, notamment par la violence envers les femmes qui le contesteraient. Au contraire, c'est l'avènement d'un nouveau modèle de genre qui s'impose.

À l'issue de ce panorama, peut-on tenter de formuler une nouvelle définition de la matrifocalité ? Pour notre part, il nous semble essentiel de nous éloigner d'une vision structuraliste et formaliste des familles matrifocales. En effet, ce n'est pas tant la structuration familiale qu'il importe de comprendre, mais la dynamique relationnelle. Cela permet de 
repérer les modes de représentation du monde, les constructions sociales des identités sexuelles et les assignations de rôles de genre et de génération qui conduisent à un système de parentalité et à une organisation familiale où l'homme, bien que reconnu dans son rôle de père et de pourvoyeur de ressources, ne parvient pas pour autant à assumer cette fonction face à la prédominance de la mère dans son propre rôle de référent central. Cette dynamique n'est pas tributaire d'une structure monoparentale, ni d'une forme matrilinéaire sur trois générations, mais elle s'inscrit dans une matrifocalité relationnelle et fonctionnelle. La matrifocalité serait alors la résultante de l'articulation de plusieurs caractéristiques, qui la distinguent fondamentalement des formes matriarcales ou monoparentales:

- Une construction sociale des identités sexuelles marquée par une forte normativité quant aux rôles féminins et masculins, et une impossibilité de remettre en question l'obligation de respectabilité des femmes et de réputation des hommes.

- Une définition des rôles parentaux fondée sur la sacralisation de la maternité dévouée, "sacrifiée " et victimaire, et sur la disqualification consensuelle des hommes dans leur rôle éducatif de pères.

- Un type de représentation du monde, des rapports intergénérationnels, des rapports sociaux de sexe et de "race» fondés aujourd'hui, pour certains acteurs sociaux, sur un imaginaire de la "dépossession", de l'«aliénation " et de la "castration ", lié au contexte post-esclavagiste dans lequel il se développe, qui ne conçoit pas de stratégies d'émancipation ni de construction des identités et des institutions créoles autres que la « résistance».

En définitive, la matrifocalité serait le système mis en place par une société meurtrie pour tenter de pallier et panser, sans le soigner, le traumatisme originel de la perte du phallus des hommes noirs.

Ce travail sur les représentations des rapports sociaux antillais dans l'imaginaire contemporain lance un vaste chantier. Il convoque non seulement une réflexion sur les bases de la société antillaise, mais appelle aussi une réflexion individuelle et collective sur les relations intimes vécues au sein des familles. Cela suppose un déplacement important dans le discours antillais : la capacité à construire une subjectivité et une individuation, afin que le sujet puisse s'exprimer à la première personne, au nom d'un "je», et ne se dilue plus dans un collectif, un "nous» qui évite de poser la question des rapports contemporains interpersonnels. 
MOTS CLÉS/KEYWORDS: matrifocalitélmatrifocality - genre/gender - famille/family patriarcat/patriarchy - créolisation/creolization - Antilles françaises/French West Indies Caraïbes/Caribbean.

BIBLIOGRAPHIE

André, Jacques

1987 L'Inceste focal dans la famille noire antillaise. Crimes, conflits, structure.

Paris, Presses universitaires de France

( Voix nouvelles en psychanalyse»).

Attias-Donfut, Claudine \& Nicole Lapierre

1997 La Famille Providence.

Trois générations en Guadeloupe. Paris,

La Documentation Française.

\section{Bastide, Roger}

1973 [1967] Les Amériques noires.

Les civilisations africaines

dans le Nouveau Monde. Paris,

Payot (« Petite bibliothèque Payot» 227).

Bell Hooks [pseud. de Gloria Jean Watkins] 1990 Yearning. Race, Gender and Cultural Politics. Boston : South End Press.

1995 Killing Rage. Ending Racism.

New York, Holt \& Co.

Bougerol, Christiane

2002 «Vivre en prison à la Guadeloupe : réputation et rivalité chez de jeunes délinquants ", Ethnologie française 32 (4) : 699-708.

\section{Bourgois, Philippe}

2001 En quête de respect. Le Crack à New York. Trad. de l'américain par Lou Aubert. Paris, Le Seuil («Liber»).

\section{Cazenave, Jaques}

1997 " Familles créoles et créolisation de la famille ", in Familles de Guadeloupe. Faits et chiffres. Pointe-à-Pitre, INSEE CAF de la Guadeloupe : 4-8.

\section{Charbit, Yves}

1987 Famille et nuptialité dans la Caraïbe. Paris, Institut national d'études démographiques-Presses universitaires de France ("Travaux et Documents - INED » 114).

Charlery, Hélène

2007 «Le patriarcat ou le féminisme noir", Revue française d'études américaines 114 (4) : 77-87.

\section{Chevannes, Barry}

1999 What We Saw and What We Reap.

Problems in the Cultivation

of Male Identity in Jamaica.

Kingston, Grace Kennedy Foundation.

\section{Chivallon, Christine}

2004 La Diaspora noire des Amériques.

Expériences et théories à partir de la Caraïbe.

Paris, CNRs Éd. («Espaces \& milieux»).

Clarke, Edith

1966 [1957] My Mother Who Fathered Me.

A Study of the Family in Three Selected

Communities in Jamaica.

London, Allen \& Unvin.

Condon, Stéphanie \& Margaret Byron

2008 «Migrations, résidence et représentations de la famille : les familles caribéennes en France et au Royaume-Uni ", Revue européenne des migrations internationales 24 (1) : 35-63

\section{Cottias, Myriam}

1990 La Famille antillaise du XVII au XIXe siècles. Etude anthropologique et démographique: enracinements créoles. Paris, École des hautes études en sciences sociales, thèse de doctorat. 
2001 «La séduction coloniale : damnation et stratégie des femmes antillaises (XVII - XIX) $)$ ", in Cécile Dauphin \& Arlette Farge, eds, Séduction et sociétés. Approches historiques. Paris, Le Seuil : 125-140.

2002 «De l'esclave à la femme "poto mitan" : mariage et citoyenneté dans les Antilles françaises (XVII - XIX ${ }^{\mathrm{e}}$ )", in Lucien Abenon, Danielle Bégot \& Jean-Pierre Sainton, eds, Construire l'histoire antillaise. Mélanges offerts à Jacques Adélaïde-Merlande. Paris : Éd. du CTHS («CTHS-histoire» 10) : 319-334.

\section{Dorlin, Elsa}

2006 La Matrice de la race. Généalogie sexuelle et coloniale de la nation française. Paris, La Découverte ("Textes à l'appui. Genre \& sexualité»).

2010 "Le mythe du matriarcat noir", in Elsa Dorlin \& Éric Fassin, eds, Reproduire le genre. Paris, Bibliothèque publique d'information, Centre Pompidou («En actes $): 69-78$.

Dorlin, Elsa, ed.

2007 Black Feminism. Anthologie du féminisme africain-américain, 1975-2000. Paris, L'Harmattan («Bibliothèque du féminisme»).

\section{Dorlin, Elsa \& Myriam Paris}

2006 «Genre, esclavage et racisme : la fabrication de la virilité" ", Contretemps 16 : 96-105.

\section{Du Bois, W. E. B.}

1899 The Philadelphia Negro.

A Social Study. Boston, Grinn \& Co.

1969 [1908] The Negro American Family.

Westport, Negro University Press.

Dubreuil, Guy

1965 «La famille martiniquaise : analyse et dynamique ", Anthropologica 7 (1) : 103-129.

\section{Fanon, Frantz}

1975 [1952] Peau noire, masques blancs. Paris, Le Seuil (« Points. Civilisation» 26).
Frazier, Edward Franklin

1973 [1939] The Negro Family

in the United States. Chicago,

University of Chicago Press.

\section{Gautier, Arlette}

1985 Les Soeurs de solitude. La condition féminine dans l'esclavage aux Antilles du XVII au XIXe siècle. Paris, Éd. caribéennes. 1986 "Politique familiale et familles monoparentales en Métropole et dans les Dom depuis 1946 ", Nouvelles Questions féministes 13: 89-100.

Giraud, Michel

1999 «Une construction coloniale de la sexualité : à propos du multipartenariat hétérosexuel caribéen ", Actes de la recherche en sciences sociales $128: 46-55$ [www.persee. $\mathrm{fr} / \mathrm{web} /$ revues/home/prescript/article/arss_0 335-5322_1999_num_128_1_3293].

Giraud, Michel et al.

1994 Analyse des comportements sexuels aux Antilles et en Guyane (ACSAG). Paris, Agence nationale de recherches sur le sida.

Glissant, Édouard

1981 Le Discours antillais. Paris, Le Seuil.

Gonzales, Nancy L.

1970 "Toward a Definition of Matrifocality ", in Norman E. Whitten Jr \& John F. Szwed, eds, Afro-American Anthropology, Contemporary Perpectives. New York, Free Press : 231-244.

\section{Gracchus, Fritz}

1980 Les Lieux de la mère dans les sociétés afro-américaines. Pour une généalogie du concept de matrifocalité.

Paris, Éd. caribéennes / Bourg-Abymes, Centre antillais de recherches et d'études.

Gutman, Herbert G.

1972 «Le phénomène invisible: la composition de la famille et du foyer noirs après la guerre de Sécession ", Annales ESC 27 (4-5) : 1197-1218 [www.persee.fr/web/revues/home/prescript/ article/ahess_0395-2649_1972_num_27 _4_422592]. 
Halfen, Sandrine

2006 Les Connaissances, attitudes, croyances et comportements face au VIH-sida aux Antilles et en Guyane en 2004.

Paris, Observatoire régional de santé d'Île de France - Agence nationale de recherches sur le sida.

\section{Herskovits, Melville J.}

1958 [1941] The Myth of the Negro Past. Boston, Beacon Press.

\section{Hill Collins, Patricia}

2000 [1990] «Defining Black Feminist Thought ", in Black Feminist Thought. Knowledge, Consciousness, and the Politics of Empowerment. London-New York, Routledge : 19-40.

\section{Kabile, Joëlle \& Clara Palmiste}

2012 «Famille, violence, citoyenneté aux Antilles françaises : pistes de réflexion ". Communication orale à la $37^{\text {th }}$ Annual Conference of the Caribbean Studies Association.

\section{Kunstadter, Peter}

1963 «A Survey of the Consanguine or Matrifocal Family ",

American Anthropologist 65 (1) : 56-66.

\section{Le Dantec-Lowry, Hélène}

\section{De l'esclave au Président.}

Discours sur les familles noires aux États-Unis. Paris, CNRs Éd.

\section{Lefaucheur, Nadine}

2012 «Genre, victimation et exclusion sociale aux Antilles françaises ", Communication orale à la $37^{\text {th }}$ Annual Conference of the Caribbean Studies Association.

Lefaucheur, Nadine, ed.

2010-2011 Pouvoirs dans la Carä̈be 17 : Genre et violences interpersonnelles à la Martinique. Schoelcher, Centre de recherche sur les pouvoirs locaux dans la Caraïbe / Paris, L'Harmattan.
Lefaucheur, Nadine \& Elizabeth Brown, eds

2011 «Relations conjugales

et configurations parentales à la Martinique ",

Politiques sociales et familiales 106 : 9-23.

Lefaucheur, Nadine \& Stéphanie Mulot

2012 «La construction et les coûts de l'injonction à la virilité en Martinique ", in Delphine Dulong, Christine Guionnet \& Érik Neveu, eds, Boys don't cry!

Les coûts de la domination masculine. Rennes, Presses universitaires de Rennes («Le sens social») : 207-230.

Lesel, Livia

1995 Le Père oblitéré. Chronique antillaise d'une illusion. Paris, L'Harmattan

( Santé, sociétés et cultures»).

Lewis, Linden

2007 «Man Talk, Masculinity,

and a Changing Social Environment",

Caribbean Review on Gender Studies 1 (1) :

1-20 [http://sta.uwi.edu/crgs/april2007/

journals/Linden_Lewis_pm_07.pdf].

Lewis, Linden, ed.

2003 The Culture of Gender and Sexuality in the Caribbean. Gainesville, University

Press of Florida.

Massé, Raymond

2008 Détresse créole. Ethnoépidémiologie de la détresse psychique à la Martinique.

Québec, Presses de l'université Laval.

Miller, Errol

1986 The Marginalization of the Black

Male. Insights from the Development

of the Teaching Profession. Kingston, Institute of Social and Economic Research.

Mintz, Sidney W.

1970 "Foreword", in Norman E. Whitten Jr \& John F. Szwed, eds, Afro-American Anthropology, Contemporary Perpectives. New York, Free Press : 1-16.

1971 "Men Women, and Trade", Comparative Studies in Social History 13 (3) : 247-269. 
Mintz, Sidney W. \& Richard Price

1976 An Anthropological Approach to the Afro-American Past. A Caribbean Perspective. Philadelphia, Institute for the Study of Human Issues.

\section{Mohammed, Patricia}

1995 "Writing Gender into History: The Negociations of Gender Relations Among Indian Men and Women in Post-Indenture Trinidad Society, 1917-1947 ", in Verene Shepherd, Bridget Brereton \& Barbara Bailey, eds, Engendering History. Caribbean Women in Historical Perspective. Kingston, Ian Randle : 20-47.

2004 «Unmasking Masculinity and Deconstructing Patriarchy : Problems and Possibilities within Feminist Epistemology ", in Rhoda Reddock, ed., Interrogating Caribbean Masculinities... : 38-67.

\section{Moynihan, Daniel P.}

1965 The Negro Family. The Case for National Action. Washington, US Government Printing, Office of Planning and Research, Department of Labor.

\section{Mulot, Stéphanie}

2000 "Je suis la mère, je suis le père!": l'énigme matrifocale. Relations familiales et rapports de sexes en Guadeloupe. Paris, École des hautes études en sciences sociales, thèse de doctorat.

2007 « Le mythe du viol fondateur aux Antilles françaises ", Ethnologie française 37 (3) : 517-524.

2008 «Chabines et métisses dans l'univers antillais : entre assignations et négociations identitaires ", CLIO. Histoire, femmes et sociétés $27: 115-134$

[http://clio.revues.org/7447].

2009 "Redevenir un homme en contexte antillais post-esclavagiste et matrifocal ", Autrepart 49 : 117-136.

2010 «Descendants d'esclaves, héritiers de la liberté : les dilemmes identitaires des Antillais aujourd'hui ", in Gilles Ferréol \& Angelina Peralva, eds, Altérité, dynamiques sociales et démocratie.

Paris, LGDJ : 109-126.

2011 Mémoires, genre, santé. Analyser la dynamique des relations sociales aux Antilles françaises. Toulouse, Université Le Mirail Toulouse 2, mémoire d'HDR.

2012 «Gender and Violence :

Theoretical and Empirical Questions from French West Indies ».

Communication orale à la $37^{\text {th }}$ Annual

Conference of the Caribbean

Studies Association.

\section{Murray, David A. B.}

1999 «Law of Desire? Race, Sexuality and Power in Male Martinican Sexual Narratives ", American Ethnologist 26 (1) : 160-172.

\section{Plummer, David, Arden McLean}

\section{\& Joel Simpson}

2008 «Has Learning Become Taboo and Is Risk-Taking Compulsory for Caribbean Boys? Researching the Relationship between Masculinities, Education and Risk", Caribbean Review of Gender Studies 2 : 1-14 [http://sta.uwi.edu/crgs/september2008/ journals/DPlummerAMcleanJSimpson.pdf].

\section{Pourette, Dolorès}

2006 Des Guadeloupéens en Île de France. Identité, sexualité, santé. Paris, Karthala ("Médecines du monde»).

\section{Reddock, Rhoda E., ed.}

2004 Interrogating Caribbean Masculinities. Theoretical and Empirical Analyses.

Mona, University of the West Indies Press.

\section{Rodman, Hyman}

1963 "The Lower-Class Value Stretch", Social Forces 42 (2) : 205-215.

\section{Rolle-Romana, Viviane}

1999 Psychothérapies d'Antillaises ensorcelées. Saint-Denis, Université Paris 8, thèse de doctorat. 
Scott, David

1997 «"An Obscure Miracle

of Connection" : Discursive Tradition

and Black Diaspora Criticism ",

Small Axe $1:$ 19-38.

Smith, Michael G.

1962 West Indian Family Structure.

Seattle, University of Washington Press.

Smith, Raymond T.

1956 The Negro Family in British Guiana.

Family Structure and Social Status

in the Villages. London, Routledge \& Paul.
West, Cornel

1993 "Black Politics,

Black Leadership : An Interview

| 9 |

with Cornel West ", The Christian Century

110 (23) : 774-777.

Wilson, Peter J.

1969 «Reputation and Respectability:

A Suggestion for Caribbean Ethnology ", Man 4 (1) : 70-84.

1973 Crab Antics. The Social Anthropology

of English-Speaking Negro Societies

of the Caribbean. New Haven,

Yale University Press.

\section{RÉSUMÉ/ABSTRACT}

Stéphanie Mulot, La matrifocalité caribéenne n'est pas un mirage créole. - La matrifocalité fait l'objet de nombreuses controverses depuis des décennies. Organisation et système relationnel familial décrits dans les sociétés caribéennes postesclavagistes, la matrifocalité a tantôt été présentée comme une spécificité, tantôt comme une illusion, voire comme un biais idéologique. Cet article propose de revenir sur les différentes analyses des relations dans les familles antillaises, et de les confronter aux résultats d'enquête récents. Il vise à montrer que, détachée des orientations politiques et idéologiques contemporaines, l'analyse anthropologique et sociologique ne peut nier l'existence d'une structuration singulière de l'imaginaire des rapports de genre, malgré sa remise en cause par des logiques sociales multiples. Cette réflexion impose de remettre en question les cadres théoriques des études sur la famille, le genre et la sexualité en contexte post-esclavagiste, et notamment le concept de patriarcat.
Stéphanie Mulot, The Caribbean Matrifocality is not a Creole Mirage. - The matrifocal families have been at the center of different controversal analysis, from decades. Described in the Caribbean societies as a familial relational system, matrifocality has either been presented as a specificity, or an illusion, or eventually an ideological biais. This article reviews different analysis on family relationships in French West Indies and the Caribbean, while facing them to the recent studies results. It aims at showing that, deprived of political and ideological contemporary perspectives, anthropology can not deny the persistance of a singular structuration of gender imaginary, even though it is questioned by many social changes. This reflexion orders to question the theoretical context of family, gender and sexuality studies, as for example the concept of patriarchy. 